\title{
Pyoverdine secreted by Pseudomonas aeruginosa as a biological recognition element for the fluorescent detection of furazolidone
}

\author{
Kun Yin ${ }^{\mathrm{a}, \mathrm{b}}$, Weiwei Zhang ${ }^{\mathrm{a}}$, Lingxin Chen ${ }^{\mathrm{a}, *}$ \\ ${ }^{a}$ Key Laboratory of Coastal Zone Environmental Processes and Ecological Remediation, Yantai Institute of Coastal Zone Research (YIC), Chinese Academy \\ of Sciences (CAS), Shandong Provincial Key Laboratory of Coastal Zone Environmental Processes, YICCAS, Yantai, Shandong 264003, PR China \\ ${ }^{\mathrm{b}}$ University of Chinese Academy of Science, Beijing 100049, PR China
}

\section{A R T I C L E I N F O}

Article history:

Received 27 May 2013

Received in revised form

17 July 2013

Accepted 22 July 2013

Available online 29 July 2013

Keywords:

Furazolidone

Pseudomonas aeruginosa strain PA1

Pyoverdine

Fluorescent detection

\begin{abstract}
A B S T R A C T
Methods for the rapid and sensitive detection of furazolidone, a pesticide used for the treatment of infections of animals and human beings, have been urgently recommended for its large residual, strong carcinogenicity and genotoxicity in the environment. In this study, a method for the detection of furazolidone based on the rapid fluorescence quenching of pyoverdine by furazolidone was developed. Pyoverdine secreted by a Pseudomonas aeruginosa strain PA1 was purified through affinity chromatography and its fluorescent property was characterized. The fluorescence of pyoverdine could be quenched by furazolidone with specificity, and based on this phenomenon a fluorescent method for furazolidone detection was established. Fluorescence of pyoverdine was quenched by furazolidone probably due to the electron transfer from pyoverdine to furazolidone. The optimal $\mathrm{pH}$ for the detection was 7.2 in $50 \mathrm{mM} 3$-(N-Morpholino) propanesulfonic acid solution, and the whole detection process could be completed within a few seconds. The linear range of the detection was $2-160 \mu \mathrm{M}$ and the limit of detection (LOD) was $0.5 \mu \mathrm{M}$. This study developed a novel fluorescent method for furazolidone detection, and the rapid and specific fluorescent biosensor can be potentially applied for furazolidone detection in the aquatic samples.
\end{abstract}

(c) 2013 Elsevier B.V. All rights reserved.

\section{Introduction}

Furazolidone [3-(5-nitrofurfurylideneamino)-2-oxazolidinone] is a kind of nitrofuran antimicrobial drugs used for stimulation of growth and treatment of bacterial and protozoal infections in poultry and aquaculture animals, to protect them from death (Ali, 1999). However, furazolidone has been proved to possess potential carcinogenic, genotoxic and mutagenic effects. Furazolidone showed strong mutagenic effect on Salmonella typhimurium and Escherichia coli strains in the Ames and SOS-chromo analyses (Gajewska et al., 1990), and could also induce the cross-linking of Vibrio cholerae DNA destroying its normal genetic function (Basak, 1995). Furthermore, furazolidone could increase mammary tumor incidence of rats when it was continuously fed to the female Sprague-Dawley rats for 2 years (Halliday et al., 1974). More importantly, furazolidone could damage the DNA of human beings' hepatoma cells, suppress cell growth, and lead to cell cycle stopped in S phase (Jin et al., 2011). Considering the harmful effects it possessed, usage of furazolidone was forbidden in food producing animals by European Union (EU) in

\footnotetext{
* Corresponding author. Tel./fax: +86 5352109130.

E-mail address: lxchen@yic.ac.cn (L. Chen).
}

1995. Later, usage of furazolidone in animals' feeds and drinking water was also banned by Ministry of Agriculture of China in 2002.

However, furazolidone is still synthesized and illegally used as feed supplement to animals in many countries, for its excellent effects on the prevention economic aquaculture animals from extensive death. For example, furazolidone is still used to treat the bacterial gastroenteritis disease in the aquatic culture zones of Jiaodong Peninsula, China. Due to its carcinogenicity, the detection of furazolidone residual in the breeding and its adjacent environment is very important for controlling its usage and environmental level. Several methods have been developed to detect furazolidone, such as HPLC (Hoogenboom et al., 1992; GaleanoDíaz et al., 1997), LC-MS (McCracken and Kennedy, 1997), LC-MS/MS (Leitner et al., 2001), and ELISA (Diblikova et al., 2005; Li J. et al., 2010). However, HPLC, LC-MS, and LC-MS/MS are time-consuming, expensive, and requirement of sophisticated equipment. ELISA is a sensitive method, but preparation of its antibody is rather trivial and time-consuming. Moreover, ELISA process could be easily affected by factors such as antigen concentration, time, temperature, and buffer system. Consequently, rapid, specific, and cost-effective method to detect furazolidone is still urgently recommended.

Pyoverdine is a yellow-green, water-soluble fluorescent pigment and belongs to a group of structurally related siderophores. Pyoverdine was firstly discovered in 1892 and its function as iron 
chelator and transporter was determined in the late 1970s by Meyer and Abdallah (1978). Chemical structure of pyoverdine is generally consisted of three parts: one is the conserved fluorescent dihydroxyquinoline chromophore; another is the acyl side chain bounded to the amino group of chromophore; and the third part is a variable peptide chain bound to the carboxyl group of chromophore (Visca et al., 2007). Pyoverdine is plentifully secreted by fluorescent Pseudomonas species only under iron-deficient conditions, and forms a very stable complex with iron ions $\left(\mathrm{Fe}^{3+}\right)$. Fluorescent Pseudomonas species use the pyoverdine related primary iron uptake system to overcome the iron limitation, depending on which they can compete with other bacteria (Fgaier and Eberl, 2010).

In this study, the pyoverdine secreted by a newly isolated $P$. aeruginosa strain PA1 was purified through affinity chromatography. Based on the fluorescence quenching of pyoverdine by furazolidone, a rapid method to quantitatively detect furazolidone was established. Furthermore, the mechanism of the fluorescence quenching of pyoverdine by furazolidone was also proposed.

\section{Materials and methods}

\subsection{Chemicals and medium}

Furazolidone, sulfadimidine, trichlorphon, sulfapyridine, sulfadoxine, sulphathiazole, bromophos methyl, diazinon, and diethylstilbestrol were obtained from Sigma (USA). Stock solutions were separately prepared by dissolving each pesticide in N, N-Dimethylformamide (DMF) or ethanol to a concentration of $5 \mathrm{mg} / \mathrm{ml}$. All other chemicals used in this study were of analytic grade. Synthetic succinate medium contained $3.0 \mathrm{~g}$ of $\mathrm{KH}_{2} \mathrm{PO}_{4}, 6.0 \mathrm{~g}$ of $\mathrm{K}_{2} \mathrm{HPO}_{4}, 0.1 \mathrm{~g}$ of $\mathrm{MgSO}_{4} \bullet 7 \mathrm{H}_{2} \mathrm{O}, 1.0 \mathrm{~g}$ of $\left(\mathrm{NH}_{4}\right)_{2} \mathrm{SO}_{4}$, and $4.0 \mathrm{~g}$ of succinic acid per liter, and the $\mathrm{pH}$ was adjusted to 7.0 using $1 \mathrm{~N} \mathrm{NaOH}$ solution before sterilization (Visca et al., 2007). Luria-Bertani (LB) medium was prepared as described by Sambrook et al. (1989).

\subsection{Instruments}

HPLC analysis was performed using Waters 600 HPLC equipped with a $4.6 \times 250 \mathrm{~nm}$ reverse-phase C18 column (Wasters, USA) in conjunction with a UV detector monitoring. Fluorescence of pyoverdine was qualitatively detected by a triple UV analyzer WFH-203B (J Link Industrial Co., Ltd., Shanghai, China), and was quantitatively measured by a FluoroMax-4 spectrofluorometer with a xenon lamp and $0.5 \mathrm{~cm}$ quartz cells. All $\mathrm{pH}$ measurements were carried out using a pH-3 meter (Lei Ci Device Works, Shanghai, China).

\subsection{Isolation and identification of pyoverdine producing bacterial strain}

Collected seawater was concentrated and plated onto LB medium amended with $1.2 \%$ agar. Pure colonies were cultured in the synthetic succinate medium for $48 \mathrm{~h}$, and the culture turned to be fluorescent was considered to be pyoverdine producing bacterium. Genomic DNA of the pyoverdine-producing bacterial strain was extracted according to the method described by Syn and Swarup (2000). The 16S rRNA gene was PCR amplified with primers 8F (5'-AGAGTTTGATCCTGGCTCAG-3') and 1492R (5'-GGTTACCTTGTTACGACTT-3') according to the method described by Lane et al. (1985). Sequencing was carried out by Sunny Bio. Co., (Shanghai, China).

\subsection{Purification of pyoverdine}

P. aeruginosa strain PA1 was inoculated into a 500-ml Erlenmeyer flask containing $150 \mathrm{ml}$ sterilized synthetic succinate medium and then incubated at $28{ }^{\circ} \mathrm{C}$ for $36 \mathrm{~h}$ in a shaker-incubator shaking at $180 \mathrm{rpm} / \mathrm{min}$. Supernatant was collected by centrifugation at 10,000g for $5 \mathrm{~min}$ at room temperature and filtered through membrane filter (pore size, $0.22 \mu \mathrm{m}$; Amicon). Pyoverdine in the supernatant was purified using Cu-sepharose (Xiao and Kisaalita, 1995). To prepare the $\mathrm{Cu}$-sepharose, $5 \mathrm{ml}$ Ni-sepharose was loaded in a syringe with a SPE cartridge (Bonna-Agela Technologies, Tianjin, China) on the bottom and was then washed with $5 \mathrm{ml}$ buffer (0.02 $\mathrm{M} \mathrm{Na}_{2} \mathrm{HPO}_{4}, 0.5 \mathrm{M} \mathrm{NaCl}$, and 0.05 M EDTA; pH 7.2). Then the column was washed with $5 \mathrm{ml}$ distilled water and incubated with $0.5 \mathrm{ml}$ of $1 \mathrm{M} \mathrm{CuSO}_{4}$. Finally, the column was washed with $5 \mathrm{ml}$ distilled water to remove the unbounded copper ions $\left(\mathrm{Cu}^{2+}\right)$. Consequently, Ni-sepharose was changed to $\mathrm{Cu}-$ sepharose.

The Cu-sepharose was washed with $5 \mathrm{ml}$ binding buffer ( $0.02 \mathrm{M} \mathrm{Na}_{2} \mathrm{HPO}_{4}$ and $1 \mathrm{M} \mathrm{NaCl}, \mathrm{pH} 7.2$ ). The supernatant containing pyoverdine was mixed with binding buffer at a ratio of $1: 1$. The mixture was loaded onto the top of the column, and the effluent was collected "drop to drop" to make sure that pyoverdine could completely contact with the $\mathrm{Cu}$-sepharose. Then the column was washed with $25 \mathrm{ml}$ binding buffer until the effluent was no longer fluorescent. Finally, the column was washed with elution buffer (0.02 $\mathrm{M} \mathrm{Na}_{2} \mathrm{HPO}_{4}$ and $1 \mathrm{M} \mathrm{NH} \mathrm{NH}_{4} \mathrm{Cl} ; \mathrm{pH}$ 7.2), and fractions were collected "drop to drop" until the color of the effluent was not green. Then $0.1 \mathrm{mM}$ EDTA was added in each fraction to recover the fluorescence (Xiao and Kisaalita, 1995). The fluorescence of each fraction was tested at emission and excitation wavelengths of $460 \mathrm{~nm}$ and $410 \mathrm{~nm}$, respectively. The fraction with the strongest fluorescence was detected by HPLC. The mobile phase was acetonitrile to $\mathrm{NaOH}$-acetic acid $(17 \mathrm{mM}, \mathrm{pH}$ 5.3), with acetonitrile gradient ranged from $0 \%$ to $100 \%$ over $40 \mathrm{~min}$ at a flow rate of $1.0 \mathrm{ml} / \mathrm{min}$. The detection was monitored at $410 \mathrm{~nm}$ under an UV detector (Hannauer et al., 2012).

\subsection{Detection of furazolidone using fluorescence quenching analysis}

Different concentrations of furazolidone, sulfadimidine, trichlorphon, sulfapyridine, sulfadoxine, sulphathiazole, bromophos methyl, diazinon, and diethylstilbestrol were separately mixed with the purified pyoverdine in the $50 \mathrm{mM}$ 3-(N-Morpholino) propanesulfonic acid (MOPS) solution to detect whether they could quench the fluorescence of pyoverdine. The purified pyoverdine in the MOPS solution without any pesticides was used as a control. The fluorescence of each solution was detected by a triple UV analyzer and a FluoroMax-4 spectrofluorometer.

\subsection{Sensitivity of the fluorescent biosensor}

To detect the linear range and limit of detection (LOD) using the fluorescent biosensor, different concentrations of furazolidone were separately added into the pyoverdine solution. The fluorescence of each mixture was measured under the optimal condition using a FluoroMax-4 spectrofluorometer equipped with a xenon lamp. Based on the Stern-Volmer equation, the fluorescent quenching efficiency in this study is calculated by $(1-\alpha) \times 100 \%$, where $\alpha$ is expressed by the ratio of fluorescence intensities before and after introduction of the furazolidone (Lakowicz, 1999).

\subsection{Detection of furazolidone in aquatic samples}

The aquatic samples were obtained from Sanyuan Lake and Phoenix Hill reservoir (Yantai, China). The aquatic samples were filtered through $0.22 \mu \mathrm{m}$ membrane to remove particulate matters. Both the original samples and furazolidone spiked samples were applied for furazolidone detection using the fluorescent biosensor and HPLC. The mobile phase of HPLC for detection of furazolidone was acetonitrile:acetate solution (acetate acid:pure water $=1: 1000$ ) 
at a ratio of $55: 45$. The flow rate was $1.0 \mathrm{ml} / \mathrm{min}$. Furazolidone was detected by a UV detector at $365 \mathrm{~nm}$. Retention time of furazolidone in the HPLC analysis was 4.06 min (Kuhne et al., 1992).

\section{Results and discussion}

\subsection{Mechanism}

The mechanism for rapid detection of furazolidone using fluorescent analyses based on the fluorescence quenching of pyoverdine by furazolidone, secreted by $P$. aeruginosa strain PA1 is shown in Scheme 1. P. aeruginosa strain PA1 grown in synthetic succinate medium could secrete the pyoverdine in a large quantity. The purified pyoverdine was obtained through affinity chromatography. Furazolidone could particularly quench the fluorescence of pyoverdine and the fluorescence quenching efficiency could be quantitatively measured using a FluoroMax-4 spectrofluorometer. Thus, the concentration of furazolidone can be quantified via the changes of its fluorescent quenching efficiency. It is well known that electron-deficient nitro compounds are strong quenchers of

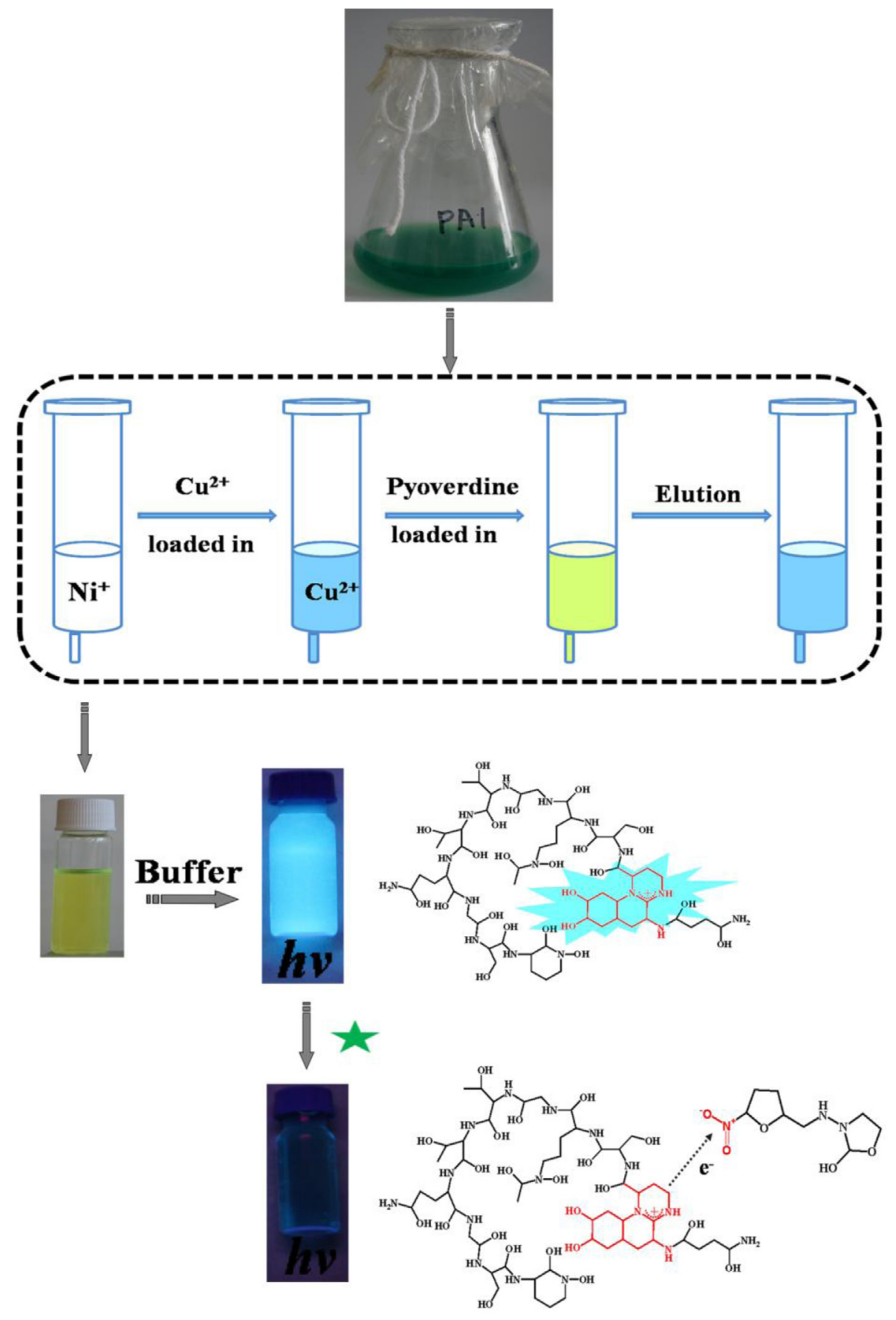


fluorophores via an electron transfer mechanism (Zhang et al., 2011; Kartha et al., 2012). Furazolidone acts as a strong electron acceptor, leading to the fluorescence quenching of an aminobenthiazole schiff based sensor, in which the formation of a nonfluorescent ground state complexes with 2-aminobenthiazole schiff base was proposed by Chen et al. (2003). Another strong fluorescence quenching of BSA by furazolidone was also observed, probably for the energy transformation from BSA to furazolidone (Li W.B. et al., 2010). In our study, the fluorescence emission spectrum of pyoverdine overlapped the absorption spectrum of furazolidone (data not shown), thus we wondered if energy transfer occurred between pyoverdine and furazolidone. The fluorescence of pyoverdine, furazolidone (at a concentration of $1 \mathrm{mM}$ ), and simultaneous presence of pyoverdine and furazolidone was measured. As shown in Fig. S1, the fluorescence of furazolidone in the presence of pyoverdine was increased. Thus, it can be postulated that the energy of pyoverdine could be transferred to furazolidone, therefore, its fluorescence intensity decreased (Selvin, 2000; Pawley, 2006).

\subsection{Identification of pyoverdine producing strain and purification of pyoverdine}

Of the strains isolated, only one strain was fluorescent after grown in synthetic succinate medium. To position the isolate within genus, the $16 \mathrm{~S}$ rRNA gene was amplified. The PCR product was purified and submitted directly for sequencing. As shown in Fig. 1, the closest relatives of the selected fluorescent strain were those of $P$. aeruginosa strains. Thus it was named $P$. aeruginosa strain PA1. P. aeruginosa strain PA1 showed strong fluorescence when grown on the solid synthetic succinate medium, which could be observed by the triple UV analyzer WFH-203B at $325 \mathrm{~nm}$. P. aeruginosa strain PA1 was grown at $28{ }^{\circ} \mathrm{C}$ in liquid synthetic succinate medium for $36 \mathrm{~h}$ to produce pyoverdine. Through affinity chromatography, the purified pyoverdine was obtained and its fluorescent character was determined. Under optimal conditions, pyoverdine showed a maximum excitation wavelength of $410 \mathrm{~nm}$ with an emission wavelength of $460 \mathrm{~nm}$.

\subsection{Detection of furazolidone using fluorescent biosensor}

The strong fluorescence of pyoverdine can be observed with naked eyes under a triple UV analyzer. As shown in Fig. 2, the fluorescence of pyoverdine was quenched immediately (within seconds), after the immediate addition of different concentrations of furazolidone. The furazolidone at concentration of $20 \mu \mathrm{M}$ could be merely distinguished by naked eyes. Traditional HPLC method was finished within about 4 min, but was nonvisible by naked eyes (Mustafa et al., 1985; Zhang et al., 2013). Compared with HPLC, furazolidone detection based on

\subsection{5}

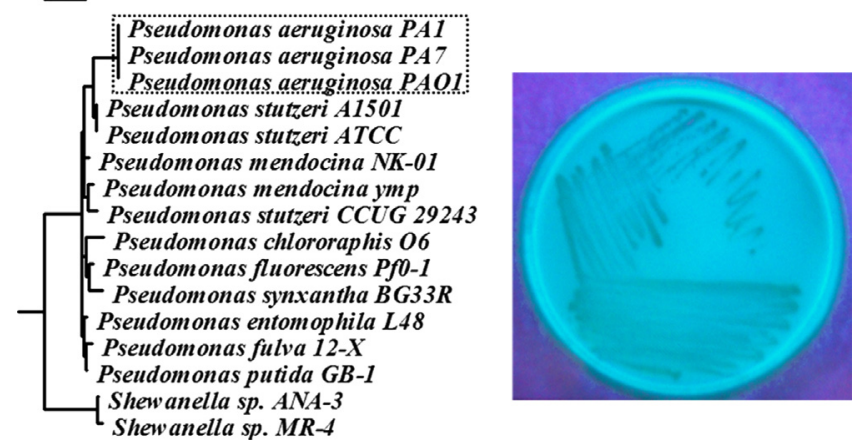

Fig. 1. Neighbor-joining phylogenetic tree with isolated bacterium $P$. aeruginosa strain PA1 and its closest relatives derived from NCBI GenBank Data Library based on 16S rRNA gene analysis (Left). The fluorescence of $P$. aeruginosa strain PA1 grown on solid synthetic succinate medium observed under the triple UV analyzer at $325 \mathrm{~nm}$ (Right).

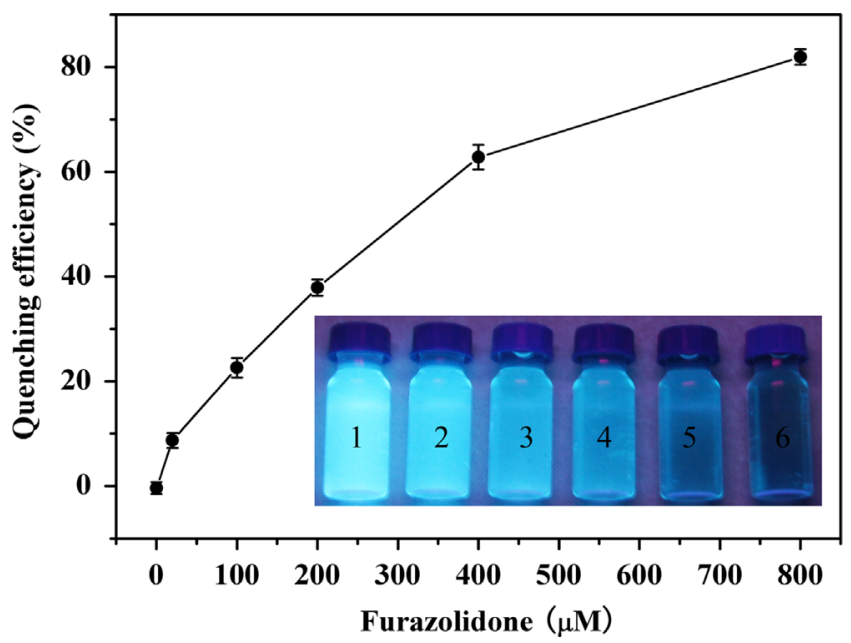

Fig. 2. Different concentrations of furazolidone were mixed with pyoverdine in the MOPS solution and were monitored under the triple UV analyzer at $325 \mathrm{~nm}$. Bottle 1 was the fluorescence of pyoverdine in the MOPS solution without furazolidone. Bottles 2, 3, 4, 5 and 6 were fluorescence of pyoverdine in the presence of 20100 , 200,400 , and $800 \mu \mathrm{M}$ of furazolidone in the MOPS solution respectively.

the fluorescent biosensor is a more rapid (within a few seconds) and visible method, and will be potentially applied for the furazolidone detection without large and sophisticated equipment.

\subsubsection{Effect of buffer on the fluorescent biosensor}

To quantitatively detect furazolidone, the optimal conditions for the fluorescent biosensor were determined. The stability of the fluorescence of pyoverdine in HEPES solution, PBS solution, $\mathrm{KH}_{2} \mathrm{PO}_{4}-\mathrm{NaOH}$ solution, $\mathrm{NaH}_{2} \mathrm{PO}_{4}$ solution, and MOPS solution was measured. The result showed that the fluorescence quenching efficiency of furazolidone is similar in different solutions. More importantly, the fluorescence of pyoverdine was the most stable in the MOPS solution, although the fluorescence intensity of pyoverdine in the MOPS solution is lower than that in other solutions. Thus, the MOPS solution was chosen as buffer system for further study.

\subsubsection{Effect of $\mathrm{pH}$ on the fluorescent biosensor}

To examine the effect of $\mathrm{pH}$ on the fluorescent biosensor, the MOPS solutions adjusted to different $\mathrm{pH}$ were used. The result showed that the fluorescence quenching efficiency of furazolidone was significantly influenced by $\mathrm{pH}$. The fluorescence quenching efficiency of furazolidone increased as $\mathrm{pH}$ rose from 4.0 to 7.2 , and then decreased once the $\mathrm{pH}$ exceeded 7.2 (Fig. S2). The highest fluorescence quenching efficiency of furazolidone was $\mathrm{pH} 7.2$, whereas the maximum fluorescence intensity of pyoverdine at was $\mathrm{pH}$ 7.0. As a reversible $\mathrm{pH}$ indicator, the fluorescence of pyoverdines was strongly $\mathrm{pH}$ dependent, and the maximum fluorescence was also observed at pH 7.0 (Elliott, 1958; Xiao and Kisaalita, 1995). But for the binding of other substrates, especially for $\mathrm{Fe}^{3+}$, the neutral or slightly alkaline $\mathrm{pH}$ was optimal condition for the formation of stable complexes (Visca et al., 2007). Considering the fact that the $\mathrm{NO}_{2}{ }^{-}$of furazolidone is also electron deficient (Morales et al., 1987; Fotouhi and Faramarzi, 2004), theoretically it was easier for furazolidone to approximate pyoverdine at slightly alkaline $\mathrm{pH}$. Thus, it was postulated that the electron transfer from pyoverdine to furazolidone could be better accomplished at pH 7.2.

\subsubsection{Effect of temperature on the fluorescent biosensor}

To examine the effect of temperature on the fluorescent biosensor, the florescence quenching efficiency of furazolidone was determined at the temperatures within the range of $0-60{ }^{\circ} \mathrm{C}$. 
The result showed that the fluorescence quenching efficiency of furazolidone showed high similarity between $0{ }^{\circ} \mathrm{C}$ and $60{ }^{\circ} \mathrm{C}$ (Fig. S3). Thus, the detection of furazolidone using the developed fluorescent biosensor could be carried out at room temperature for convenience.

\subsubsection{Effect of incubation time on the fluorescent biosensor}

To determine the effect of incubation time on the fluorescent biosensor, furazolidone was incubated with pyoverdine in the MOPS solution ( $\mathrm{pH}$ 7.2) for different time intervals. The result showed that the fluorescence remained stable instantaneously once the quenching effects happened, which means that the detection process can be completed within $1 \mathrm{~min}$ (Fig. S4). The developed fluorescent biosensor was the most rapid method for detection of furazolidone.

\subsection{Specificity of furazolidone detection by fluorescent biosensor}

To examine the specificity of furazolidone detection by the fluorescent biosensor, furazolidone, as well as sulfadimidine, trichlorphon, sulfapyridine, sulfadoxine, sulphathiazole, bromophos methyl, diazinon, and diethylstilbestrol, was separately applied to the pyoverdine MOPS solution. The purified pyoverdine in the MOPS solution without any pesticide was used as a control. The concentration of furazolidone was $100 \mu \mathrm{M}$, while the concentrations of other pesticides were 10-fold concentration of furazolidone. As shown in Fig. 3a, $25 \%$ of the fluorescence of pyoverdine was quenched by furazolidone, but the fluorescence of pyoverdine in the presence of other pesticides still remained as that of control. And the phenomenon of fluorescence quenching could be observed with naked eyes under the triple UV analyzer. Thus, it was concluded that the fluorescent biosensor specifically responded to furazolidone.

To detect the fluorescence quenching effect of furazolidone simultaneous in the presence of other pesticides, furazolidone and other pesticides were simultaneously added into the reaction system. The blank was pyoverdine alone in the MOPS solution. The concentrations of other interfering substrates were 10 -fold of the concentration of furazolidone. The result showed that in the presence of the interfering pesticides, the fluorescence quenching efficiency of furazolidone remained the same as that of furazolidone alone (Fig. 3b). Thus, it was concluded that the fluorescent biosensor responded to furazolidone without the interference of other pesticides. Although fluorescent biosensor owns advantages for its simple preparation, high specificity, biocompatibility, and less poisonousness, only a few methods for pesticides detection based on fluorescent analyses have been developed (Hai et al., 2013; Xing et al., 2013). Moreover, few fluorescent sensors for the detection of pesticides by biological recognition element were established. Hence, the fluorescent biosensor was a novel strategy to detect furazolidone, which could be potentially applied in the aquatic samples.

\subsection{Sensitivity of furazolidone detection by fluorescent biosensor}

To determine the sensitivity of the fluorescent biosensor, the concentrations of furazolidone ranging from 2 to $1000 \mu \mathrm{M}$ was applied to the fluorescent analyses under the optimal condition. The fluorescence intensity of pyoverdine decreased as the concentrations of furazolidone increased. As shown in Fig. 4, the fluorescent quenching efficiency of furazolidone was linear within the range of $2-160 \mu \mathrm{M}$, which was comparable to other earlier reported biosensor (Kumar and D'Souza, 2010). The LOD of the fluorescent biosensor was $0.5 \mu \mathrm{M}$ [signal-to-noise $(S / N)$ ratio=3], which was also comparable to a carbon nanotube/organophosphorus hydrolase electrochemical biosensor (Deo et al., 2005) and amperometric microbial biosensor for direct determination of organophosphate pesticides (Mulchandani et al., 2001).

\subsection{Detection of furazolidone using fluorescent biosensor in aquatic samples}

The fluorescent biosensor based on the fluorescence quenching of pyoverdine by furazolidone was proved to be efficient in our initial laboratory experiment. To investigate whether furazolidone in the aquatic samples can be quantitatively detected out, lake water and reservoir water were used as matrices. Furazolidone was not detected in these real samples both using HPLC and fluorescent analyses. Thus, furazolidone was spiked into the samples followed by adjusting $\mathrm{pH}$ to 7.2 with the MOPS solution. Then, the furazolidone detection using the developed fluorescent biosensor and HPLC was carried out (Table 1). The recoveries of spiked samples were ranged from $94.8 \%$ to $114.5 \%$ using the fluorescent biosensor, and the similar result was also obtained using HPLC with the recoveries ranged from $102.3 \%$ to $116.0 \%$. This result indicated that the fluorescent biosensor could quantitatively detect furazolidone in aquatic samples. Concluded these results indicated that the fluorescent biosensor has the potential to be applied in the rapid furazolidone detection with high specificity.
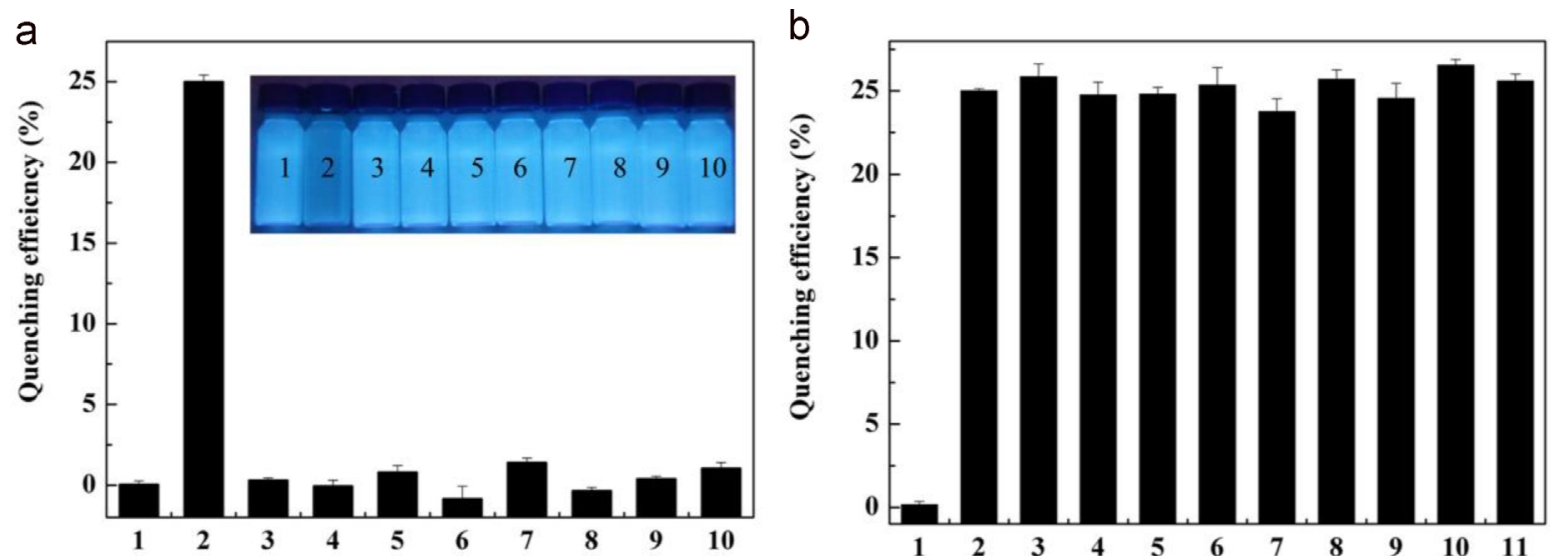

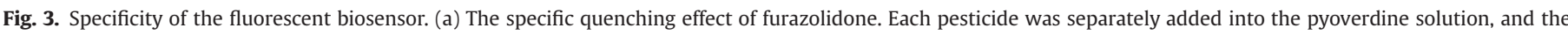

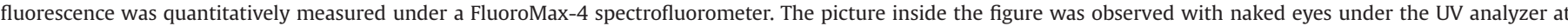

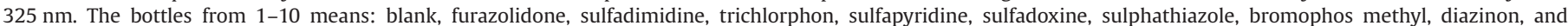

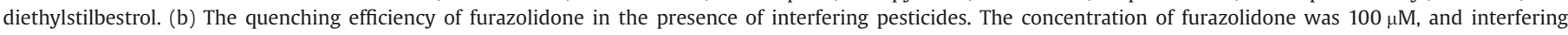

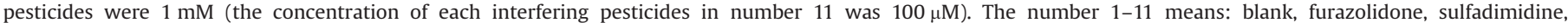
trichlorphon, sulfapyridine, sulfadoxine, sulphathiazole, bromophos methyl, diazinon, diethylstilbestrol, and all interfering pesticides together. 

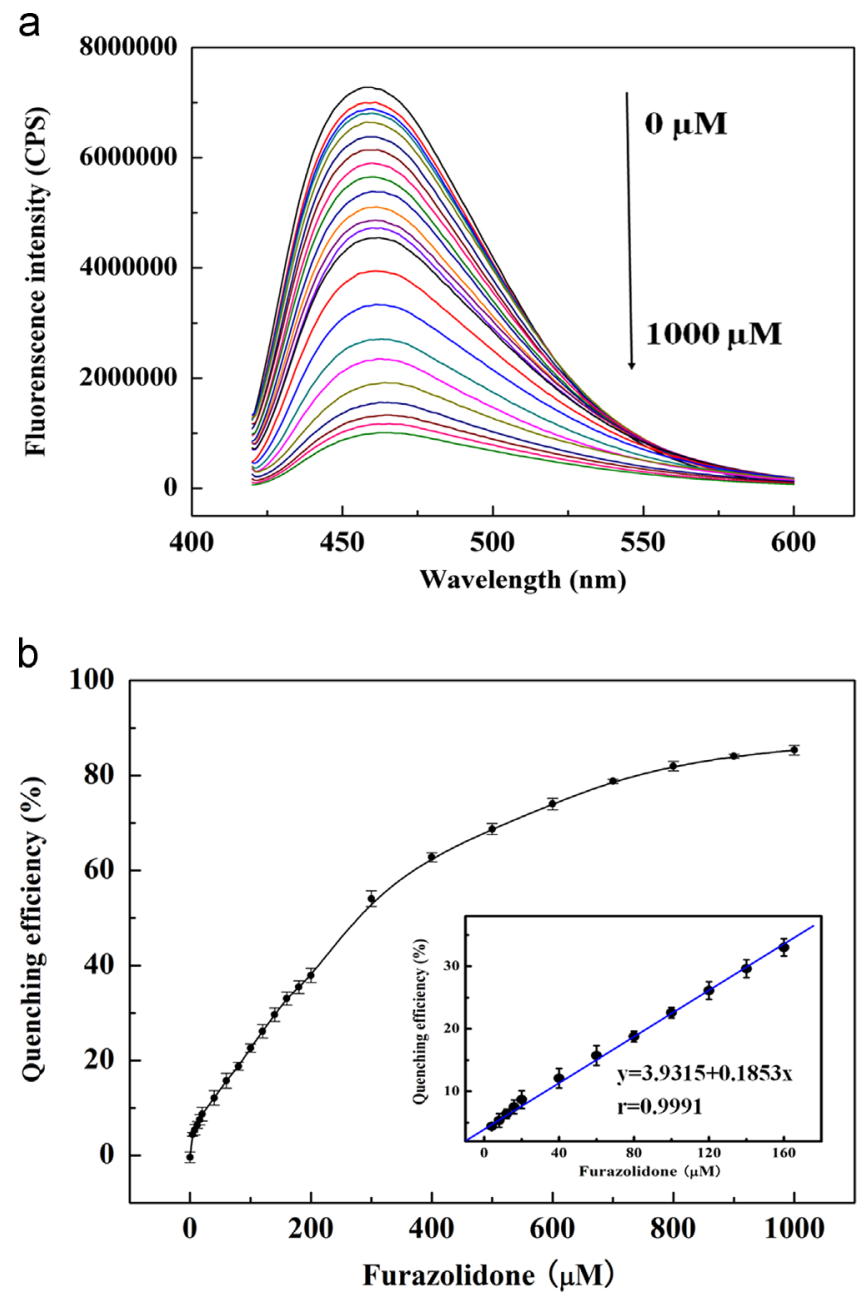

Fig. 4. Linear range and LOD of the fluorescent biosensor. (a) Fluorescent emission spectra of pyoverdine in the presence of furazolidone at concentrations of $0,4,8$, $12,16,20,40,60,80,100,200,300,400,500,600,700,800,900$, and $1000 \mu \mathrm{M}$. (b) Detection of furazolidone using fluorescent biosensor (inset: linearity between 2 and $160 \mu \mathrm{M}$ with liner regression equation, $y=3.9315+0.1853 x$ and $r=0.9991$ where ' $y$ ' represents the quenching efficiency and ' $x$ ' represents the furazolidone concentration)

Table 1

The measurement of spiked furazolidone in lake water and reservoir water and the recovery by fluorescent biosensor and HPLC. Data are the means for three independent experiments and are presented as the means $\pm \mathrm{SE}$.

\begin{tabular}{clllll}
\hline Sample & $\begin{array}{l}\text { Added } \\
\text { conc. } \\
(\mu \mathrm{M})\end{array}$ & $\begin{array}{l}\text { Detected conc. } \\
(\mu \mathrm{M}) \\
\text { (biosensor) }\end{array}$ & $\begin{array}{l}\text { Recovery } \\
(\%) \\
\text { (biosensor) }\end{array}$ & $\begin{array}{l}\text { Detected } \\
\text { conc. }(\mu \mathrm{M}) \\
(\mathrm{HPLC})\end{array}$ & $\begin{array}{l}\text { Recovery } \\
(\%)(\mathrm{HPLC})\end{array}$ \\
\hline Lake & 20 & $22.3 \pm 1.6$ & $111.5 \pm 8.0$ & $21.2 \pm 0.2$ & $106.0 \pm 1.0$ \\
water & 40 & $41.3 \pm 2.4$ & $103.3 \pm 6.0$ & $40.9 \pm 0.3$ & $102.3 \pm 1.5$ \\
& 60 & $60.2 \pm 3.2$ & $100.3 \pm 5.3$ & $61.7 \pm 0.2$ & $102.8 \pm 1.0$ \\
Reservoir & 20 & $20.6 \pm 2.7$ & $103.0 \pm 13.5$ & $23.2 \pm 0.1$ & $116.0 \pm 0.5$ \\
water & 40 & $39.6 \pm 1.9$ & $99.0 \pm 4.8$ & $44.6 \pm 0.3$ & $111.5 \pm 1.5$ \\
& 60 & $56.9 \pm 2.3$ & $94.8 \pm 3.8$ & $61.5 \pm 0.2$ & $102.5 \pm 1.0$ \\
\hline
\end{tabular}

\section{Conclusions}

An efficient fluorescent biosensor based on fluorescence quenching of pyoverdine by furazolisone was developed for the detection of furazolidone. Pyoverdine was purified from a fluorescent $P$. aeruginosa strain PA1, and exhibited the maximum excitation wavelength of $410 \mathrm{~nm}$ with emission wavelength of $460 \mathrm{~nm}$. The fluorescence quenching of pyoverdine may be due to the electron transfer from pyoverdine to furazolidone. This fluorescent biosensor owns outstanding specificity and sensitivity in furazolidone detection. A linear range of $2-160 \mu \mathrm{M}$, and a LOD of $0.5 \mu \mathrm{M}$ was established using the fluorescent biosensor for furazolidone detection. More importantly, the quenching reaction completes in seconds, which suggests that the fluorescent biosensor is also time-efficient. Therefore, the fluorescent biosensor owns outstanding advantages over other detection methods and may be potentially applied for furazolidone detection in the aquatic samples.

\section{Acknowledgments}

The authors gratefully thank the financial support provided by the Innovation Projects of the Chinese Academy of Sciences Grant KZCX2-EW-206, the National Natural Science Foundation of China Grant 31200041 and 21275158, the Scientific Research Foundation for the Returned Overseas Chinese Scholars, State Education Ministry, and the 100 Talents Program of the Chinese Academy of Sciences.

\section{Appendix A. Supporting information}

Supplementary data associated with this article can be found in the online version at http://dx.doi.org/10.1016/j.bios.2013.07.038.

\section{References}

Ali, B.H., 1999. Veterinary Research Communications 23, 343-360.

Basak, J., 1995. Mutation Research/Fundamental and Molecular Mechanisms of Mutagenesis 327, 5-15.

Chen, L.X., Niu, C.G., Zeng, G.M., Zeng, G.M., Shen, G.L., Yu, R.Q., 2003. Analytical Letters 36, 2609-2622.

Deo, R.P., Wang, J., Block, I., Mulchandani, A., Joshi, K.A., Trojanowicz, M., Scholz, F., Chen, W., Lin, Y., 2005. Analytica Chimica Acta 530, 185-189.

Diblikova, I., Kevin, M., Cooper, D., Glenn, K., Milan, F., 2005. Analytica Chimica Acta 540, 285-292.

Elliott, R.P., 1958. Applied Microbiology 6, 241-246.

Fgaier, H., Eberl, H.J., 2010. Journal of Theoretical Biology 263, 566-578.

Fotouhi, L., Faramarzi, S., 2004. Journal of Electroanalytical Chemistry 568, 93-99.

Gajewska, J., Szczypka, M., Tudek, B., Szymczyk, T., 1990. Mutation Research/ Fundamental and Molecular Mechanisms of Mutagenesis 232, 191-197.

GaleanoDíaz, T., Guiberteau Cabanillas, A., Acedo Valenzuela, M.I., Correa, C.A., Salinas, F., 1997. Journal of Chromatography A 64, 243-248.

Hai, N.N, Chinh, V.D., Thuy, U.T.D., Chi, T.K., Yen, N.H., Cao, D.T., Liem, N.Q., Nga, P.T., 2013. International Journal of Nanotechnology 10, 137-145.

Halliday, R.P., Sutton, M.L., Sigler, F.W., 1974. Submitted to WHO by Smith Kline Beecham Animal Health. West Chester, PA, USA.

Hannauer, M., Schäfer, M., Hoegy, F., Gizzi, P., Wehrung, P., Mislin, G.L.A., Budzikiewicz, H., Schalk, I.J., 2012. FEBS Letters 586, 96-101.

Hoogenboom, L.A.P., Berghmans, M.C.J., Polman, T.H.G., Parker, R., Shaw, I.C., 1992. Food Additive Contaminants 9, 623-630.

Jin, X., Tang, S.S., Chen, Q., Zou, J.J., Zhang, T., Liu, F.Y., Zhang, S., Sun, C.D., Xiao, X.L., 2011. Toxicology Letters 201, 205-212.

Kartha, K.K., Babu, S.S., Srinivasan, S., Ajayaghosh, A., 2012. Journal of the American Chemical Society $134,4834-4841$.

Kuhne, M., Kobe, A., Fries, R., Ebrecht, A., 1992. Archiv für Lebensmittelhygiene 43, 59-63.

Kumar, J., D’Souza, S.F., 2010. Biosensors and Bioelectronics 26, 1292-1296.

Lakowicz, J.R. (Ed.), 1999. Principles of Fluorescence Spectroscopy. Kluwer Academic/Plenum Publishers, New York.

Lane, D., Pace, B., Olsen, G., Stahl, D., Sogin, M., Pace, N., 1985. Proceedings of the National Academy of Sciences of the United States of America 82, 6955-6959. Leitner, A., Zöllner, P., Lindner, W., 2001. Journal of Chromatography A 939, 49-58.

Li, J., Liu, J., Zhang, H.C., Li, H., Wang, J.P., 2010. Analytica Chimica Acta 678, 1-6.

Li, W.B., Zhang, G.W., Pan, J.H., Zhao, N., 2010. Journal of Nanchang University (Natural Science), 566-570.

McCracken, R.J., Kennedy, D.G., 1997. Journal of Chromatography B: Biomedical Sciences and Applications 691 (1), 87-94.

Meyer, J.M., Abdallah, M.A.J., 1978. General Microbiology 107, 319-328.

Morales, A., Richter, P., Toral, M., 1987. Analyst 112, 965-970.

Mulchandani, P., Chen, W., Mulchandani, A., Wang, J., Chen, L., 2001. Biosensors and Bioelectronics 16, 433-437. 
Mustafa, A.I., Ali, B.H., Satti, A.M., 1985. Comparative Biochemistry and Physiology Part C: Comparative Pharmacology 81, 167-169.

Pawley, J.B. (Ed.), 2006. Handbook of Biological Confocal Microscopy. Springer, New York, NY.

Sambrook, J., Fritsch, E.F., Maniatis, T., 1989. Cold Spring Harbor, NY. vol. 6, pp. 179187.

Selvin, P.R., 2000. Nature Structural Biology 7, 730-734.

Syn, C.K., Swarup, S., 2000. Analytical Biochemistry 278, 86-90.
Visca, P., Imperi, F., Lamont, I.L., 2007. Trends in Microbiology 15, 22-30.

Xiao, R., Kisaalita, W.S., 1995. Applied and Environmental Microbiology 61, 3769-3774.

Xing, X.Q., Zhou, Y.Y., Sun, J.Y., Tang, D.B., 2013. Analytical Letters 46, 694-705. Zhang, K., Zhou, H.B., Mei, Q.S., Wang, S.H., Guan, G.J., Liu, R.Y., Zhang, J., Zhang, J.P., 2011. Journal of the American Chemical Society 133, 8424-8427.

Zhang, W.W., Niu, Z.L., Yin, K., Liu, F., Chen, L.X., 2013. International Biodeterioration and Biodegradation 77, 45-50. 